\title{
EXTRACTION OF FERROMAGNETIC PARTICLES BY A SUSPENDED MAGNET
}

\section{J. SVOBODA}

Magnetic Technology Consultants (Pty) Ltd., P.O. Box 598, Vereeniging 1930, South Africa

(Received October 30, 1992)

\begin{abstract}
This paper investigates the mechanisms of extraction of ferromagnetic bodies from a stream of bulk material by suspended magnets. It is shown that the force density, usually used to evaluate a suspended magnet, can give erroneous criteria for the magnet design and that the magnetic field strength is the primary parameter in evaluating a suspended magnet.
\end{abstract}

\section{INTRODUCTION}

Suspended magnetic separators have been used over many decades to improve material purity and to protect processing machinery by removing unwanted iron and steel objects from conveyed bulk material.

Although the design procedures of such magnetic systems that use either electromagnets or permanent magnets are well established, the understanding of fundamental principles of extraction of metal is inadequate and standards for selecting suitable separators are inconsistent and often incorrect.

The common practice $[1,2]$ is to base the evaluation of a magnetic separator on the so-called "force index" which is essentially the magnetic force density acting on a ferromagnetic body to be removed from a stream of material. Such an approach requires that the product of the magnetic induction and the gradient of the magnetic field assumes a certain minimum value for the extraction to occur. 
Although this concept is valid for certain special shapes of bodies to be removed, it does not, in general, describe correctly the behaviour of ferromagnetic bodies in a magnetic field and can lead to erroneous design of a magnetic circuit.

The present paper is concerned with the development of a model of the behaviour of ferromagnetic bodies under the influence of the magnetic field generated by a suspended magnet. The comparison with the "force index" approach will be made in terms of magnetic field pattern, body shape and size and the depth of the burden of material conveyed on a belt.

\section{FERROMAGNETIC PARTICLE IN A MAGNETIC FIELD}

\section{Magnetic Force}

A starting point in the evaluation of the efficiency of a suspended magnet is the requirement that the magnetic force $F_{\mathrm{m}}$ acting on a body be greater than the sum of competing forces, e.g. force of gravity $F_{\mathrm{g}}$, the environmental drag $F_{\mathrm{d}}$, force of friction $F_{\mathrm{f}}$ etc.

The magnetic force can be written as:

$$
\mathbf{F}_{m}=(\mathbf{m} \cdot \nabla) \mathbf{B}_{0}
$$

where $B_{0}$ is the external magnetic induction and $\boldsymbol{m}$ is the effective magnetic dipole moment of the body.

When both the body and the external medium are homogeneous, isotropic and linear, and the body has an ellipsoidal shape, the effective dipole moment is [3]:

$$
\mathbf{m}=\mathrm{V} \mathbf{H}_{0} \frac{\mu_{1}-\mu_{2}}{\mu_{2}+\mathrm{D}\left(\mu_{1}-\mu_{2}\right)}
$$

where $V$ is the volume of the body, $\boldsymbol{H}_{\mathbf{0}}$ is the external magnetic field strength, $\mu_{1}$ and $\mu_{2}$ are the relative magnetic permeabilities of the body and of the external medium, respectively, $D$ is the demagnetization factor appropriate for the axis of the ellipsoid along which $\boldsymbol{H}_{0}$ is directed. 
Using eqs.(1) and (2) we get for the magnetic force:

$$
\mathbf{F}_{\mathrm{m}}=\left[\frac{1}{4 \pi}\right] \mathrm{V} \frac{\mu_{1}-\mu_{2}}{\mu_{2}+\mathrm{D}\left(\mu_{1}-\mu_{2}\right)}\left(\mathbf{H}_{0} \cdot \operatorname{grad}\right) \mathbf{B}_{0}
$$

When the square bracket is included, the cgs system is understood, otherwise the SI system is applicable.

For a ferromagnetic body in a non-magnetic or weakly magnetic medium we have $\mu_{1} \gg 1$ and $\mu_{2} \doteq 1$, so that eq. (3) becomes:

$$
\mathbf{F}_{\mathrm{m}}=\left[\frac{\mu_{0}}{4 \pi}\right] \mathrm{V} \frac{\mu_{1}-1}{\mu_{0}\left(1+\mathrm{D}\left(\mu_{1}-1\right)\right)}\left(\mathbf{B}_{0} \cdot \operatorname{grad}\right) \mathbf{B}_{0}
$$

The relative magnetic permeability of the body $\mu_{1}$ is the function of the magnetic field inside the body, $\mu_{1}=\mu_{1}(\mathrm{H})$, where [3]:

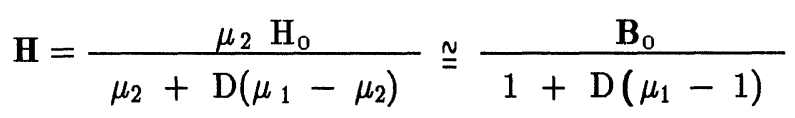

for $\mu_{2} \cong 1$.

$D$ is the demagnetization factor appropriate for the axis of the easiest magnetization (i.e. the axis of the smallest $D$ ), as the body is assumed to be oriented so that this axis is parallel to the external field $H_{0}$.

The expression (4) for the magnetic force is a product of two factors: the first factor reflects the physical properties of particles to be removed, the second factor, the magnetic force density

$$
f_{m}=B_{0} \cdot \operatorname{grad} B_{0}
$$

describes the properties of the magnetic system.

It is this magnetic force density that many designers and engineers call "force index" and use to evaluate magnetic separators. 
Let us assume that a particle to be extracted by a suspended magnet rests on a stationary conveyor and no burden of bulk material is present. In equilibrium $F_{\mathrm{m}}=$ $F_{\text {g, where }}$

$$
\mathbf{F}_{\mathbf{g}}=\mathrm{V}\left(\rho_{\mathrm{p}}-\rho_{\mathrm{m}}\right) \mathbf{g}
$$

where $g$ is the gravitational acceleration, $\rho_{\mathrm{p}}$ is the density of the body and $\rho_{\mathrm{m}}$ is the density of medium. We shall assume that $\rho_{\mathrm{p}} \gg \rho_{\mathrm{m}}$ and thus:

$$
\mathbf{f}_{\mathrm{m}}=\left[\frac{4 \pi}{\mu_{0}}\right] \rho_{\mathrm{p}} \frac{\left(1+\mathrm{D}\left(\mu_{1}-1\right)\right) \mu_{0}}{\mu_{1}-1}
$$

Eq. (8) thus determines the minimum force density (or force index) required to lift a stationary ferromagnetic body of relative magnetic permeability $\mu_{1}$, density $\rho_{\mathrm{p}}$ and demagnetization factor $D$, from its position on a belt, in the absence of burden of bulk material.

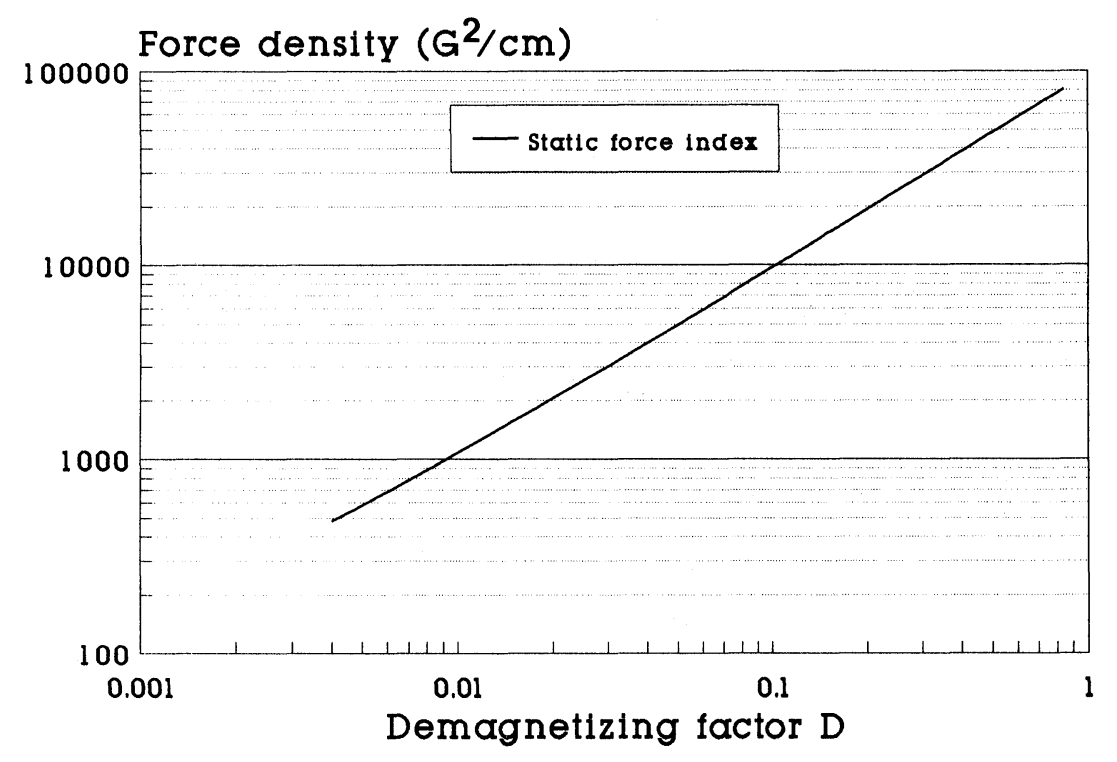

Fig. 1. Magnetic force density (or force index), for steel, as a function of demagnetization factor. 
It can be seen that this minimum magnetic force density is independent of particle size and depends solely on its shape (through the demagnetization factor), its magnetic properties (through the relative magnetic permeability) and its density. Eq. (8) also shows that the ratio of force indices for various shapes is in the ratio of their demagnetization factors (provided $D\left(\mu_{\mathrm{r}}-1\right) \gg 1$, which is satisfied for all shapes but very long rods or thin plates, for which $D \ll 1$ ). Table I gives several examples of ratios of force index.

The minimum static force index (or magnetic force density) as a function of demagnetization factor, for a given material characterized by $\rho_{\mathrm{p}}$ and $\mu_{\mathrm{r}}$, in the absence of the burden thus has general validity for any size of the object to be lifted. Figure 1 shows such a dependence for steel $\left(\rho_{\mathrm{p}}=7.8 \mathrm{~g} \mathrm{~cm}^{-3}, \mu_{\mathrm{r}}=1000\right)$. It can be seen that, for instance, for a steel sphere $(D=0.33) f_{m}=31.8 \times 10^{3} G^{2} \mathrm{~cm}^{-1}$, or $80.8 \times 10^{3} \mathrm{G}^{2} \mathrm{inch}^{-1}$ ).

Table I. Static force indices and their ratios for selected shapes

\begin{tabular}{|c|c|c|c|}
\hline Object & $\ell / d$ & $\begin{array}{l}\text { Force index FI } \\
\qquad\left(\mathrm{G}^{2} \mathrm{~cm}^{-1}\right) \\
\left(\times 10^{-6} \mathrm{~T}^{2} \mathrm{~m}^{-1}\right)\end{array}$ & $\begin{array}{c}\text { Ratio } \\
\text { FI/FI }\end{array}$ \\
\hline Rod & $\begin{array}{r}1.0 \\
4.0 \\
10.0 \\
100.0\end{array}$ & $\begin{array}{r}29,889 \\
9,515 \\
4,037 \\
, 481\end{array}$ & $\begin{array}{l}7.4 \\
2.4 \\
1.0 \\
0.12\end{array}$ \\
\hline Sphere & & 31,811 & 7.9 \\
\hline $\begin{array}{c}\text { Plate } \\
13 \times 50 \times 50 \mathrm{~mm} \\
13 \times 200 \times 200 \\
25 \times 200 \times 200\end{array}$ & & $\begin{array}{l}20,300 \\
11,130 \\
15,300\end{array}$ & $\begin{array}{l}5.0 \\
2.8 \\
3.8\end{array}$ \\
\hline
\end{tabular}

For majority of objects that are encountered in practice the assumption that the external magnetic field is directed along the axis of easy magnetization of the body is invalid. Objects such as plates, rods or bolts usually rest on a conveyor belt with their axes of easy magnetization perpendicular to the direction of the external 
magnetic field. Demagnetization factors appropriate for such orientations are high and large force densities would be required to lift the body towards the magnet.

\section{Torque on a Magnetizable Body}

Under the above mentioned circumstances, the role of the magnet should be first to re-orient the ferromagnetic body in such a way that its long axis (the axis of easy magnetization) is parallel with the direction of the external magnetic field. This is accomplished by exerting a torque on the body by the externally applied magnetic field.

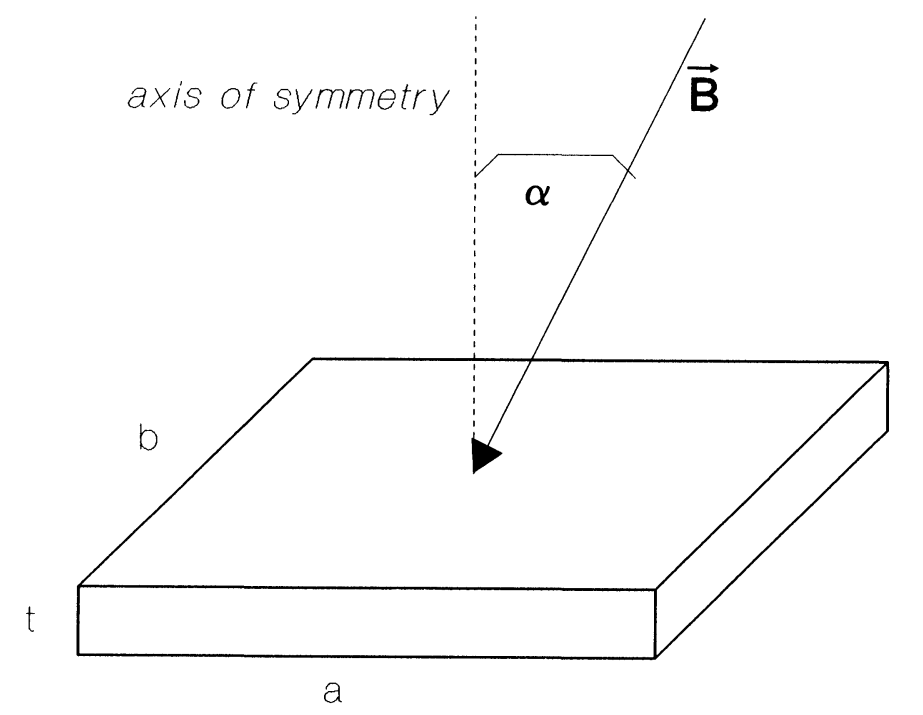

Fig. 2. Ferromagnetic plate in a transverse external magnetic field

The magnetic torque $\mathbf{T}$ is given $[3,4]$ by:

$$
\mathbf{T}=\mathbf{m} \times \mathbf{B}_{0}
$$

where $\boldsymbol{m}$ is the magnetic moment of the particle and $\mathbf{B}_{0}$ is the magnetic induction of the external magnetic field. Since $\mathbf{m}=\mathrm{VM}$ where $\boldsymbol{M}$ is the magnetization of the body, eq. (9) can be rewritten : 


$$
\mathbf{T}=\mathbf{V} \mathbf{M} \times \mathbf{B}
$$

Assuming a body of a spheroidal shape, the torque is perpendicular to the plane passing through the axis of symmetry of the body and the direction of $\boldsymbol{B}_{0}$ (the induced magnetic dipole moment $\boldsymbol{m}$ lies in this plane), the magnitude of the torque is given by [5] (by analogy with the torque on a dielectric spheroid in an electric field):

$\mathrm{T}=\frac{\left(\mu_{1}-\mu_{2}\right)^{2}|1-3 \mathrm{D}| \mathrm{H}_{0} \mathrm{~B}_{0} \mathrm{~V} \sin 2 \alpha}{[4 \pi] 2\left(\left(\mu_{2}+\mathrm{D}\left(\mu_{1}-\mu_{2}\right)\right)\left(\left(1+\mu_{1}\right) \mu_{2}-\mathrm{D}\left(\mu_{1}-\mu_{2}\right)\right)\right.}$

and, for $\mu_{2} \cong 1$

$\mathrm{T}=\frac{\left(\mu_{1}-1\right)^{2}|1-3 \mathrm{D}| \mathrm{B}_{0}^{2} \mathrm{~V} \sin 2 \alpha}{[4 \pi] 2\left(1+\mathrm{D}\left(\mu_{1}-1\right)\right)\left(1+\mu_{1}-\mathrm{D}\left(\mu_{1}-1\right)\right)}$

Here $\alpha$ is the angle between the direction of $B_{0}$ and the axis of symmetry of the spheroid, and $D$ is the demagnetization factor along this axis. The torque is directed so that it tends to turn the axis of symmetry of a prolate (i.e. $\mathrm{D}<0.33$ ) or oblate $(\mathrm{D}>0.33)$ spheroid parallel or perpendicular to $B_{0}$, respectively. Thus the torque tends to align the axis of easy magnetization of the body along $\boldsymbol{B}_{0}$.

Equation (12) neglects the contribution of terms due to $\operatorname{grad} \boldsymbol{B}_{0}$ to the torque, but this should be an adequate approximation under most circumstances. It can be seen that the torque is maximized when $\alpha=45^{\circ}$ so the geometry with such an angle should be strived for.

In equilibrium, the magnetic torque is balanced by the torque exerted by the weight of the body $G_{\mathrm{p}}$ and by the weight of the burden $G_{\mathrm{b}}$. We can thus write for a plate:

$$
\mathrm{T} / \mathrm{V}=\frac{1}{2} \mathrm{~g} \mathrm{a}\left[\rho_{\mathrm{p}}+\mathrm{h} \rho_{\mathrm{b}} / \mathrm{t}\right]
$$

where $a$ and $t$ are dimensions of the plate as shown in Fig. 2, $h$ is the height of the burden of the material and $\rho_{\mathrm{b}}$ is its bulk density. 
Combining eqs. (12) and (13) we obtain:

$\mathrm{B}_{0}^{2} \sin 2 \alpha=[4 \pi] \operatorname{ag}\left(\rho_{\mathrm{p}}+\frac{\mathrm{h}}{\mathrm{t}} \rho_{\mathrm{b}}\right) \frac{\left[\frac{1}{\mu_{1}-1}+\mathrm{D}\right]\left[\frac{\mu_{1}+1}{\mu_{1}-1}-\mathrm{D}\right]}{|1-3 \mathrm{D}|}$

For $\mu_{1} \gg 1$ we then have:

$\mathrm{B}_{0}^{2} \sin 2 \alpha \stackrel{\cong}{=}[4 \pi] \mathrm{ag}\left[\rho_{\mathrm{p}}+\frac{\mathrm{h}}{\mathrm{t}} \rho_{\mathrm{b}}\right] \frac{\mathrm{D}(1-\mathrm{D})}{|1-3 \mathrm{D}|}$

It can be seen that if the magnetic system generates the field that is perpendicular to the plane of the plate, it will not orient itself since $\alpha=90^{\circ}$ and thus $\mathbf{T}=0$. Also, it transpires from eq. (10) that the gradient of the magnetic field does not contribute, in the first approximation, to the torque. Thus, the magnitude of the field determines whether the reorientation of the body occurs or not.

It is thus clear that in contrast to the usual description by force index which demands that the magnetic system be designed in such a way that $(\boldsymbol{B} \cdot \operatorname{grad} \boldsymbol{B})$ achieves a certain minimum value, the present physical picture requires a minimum value of magnetic field only. The physical content of such an conclusion can have important implications for the design of the magnetic system.

It follows from the previous analysis that in a suspended magnet, an important parameter is the magnetic field strength. If the field strength is high enough so that it can orient the body along the axis of easy magnetization, the magnitude of the magnetic force density $(\boldsymbol{B} \cdot \operatorname{grad} \boldsymbol{B})$ is required to be high enough to lift the body in the direction of its axis of easy magnetization, only. Therefore, the force index is not the only parameter for the evaluation of a suspended magnet.

For instance, the force index $(\boldsymbol{B} \cdot \operatorname{grad} \boldsymbol{B})$ may be just large enough to lift an object in the direction of its axis of easy magnetization, but the magnitude of the magnetic field itself may still be too low so that the condition of eq. (15) will not be fulfilled and the object will not re-orient itself appropriately in order to be lifted. 
When designing the magnetic system, it is thus necessary to ensure that the magnitude of $\boldsymbol{B}_{\mathbf{0}}$ and its direction satisfy eq. (15). An additional design condition is that the force density satisfies eq. (8), with $D$ representing the demagnetization factor along the axis of easy magnetization.

Typical dependence of the minimum magnetic field on the depth of the burden, for selected sizes of steel rods and plates is shown in Figs. 3 and 4, respectively. Similar diagrams can be constructed for other shapes and sizes using eq.(14), provided the demagnetization factors are known.

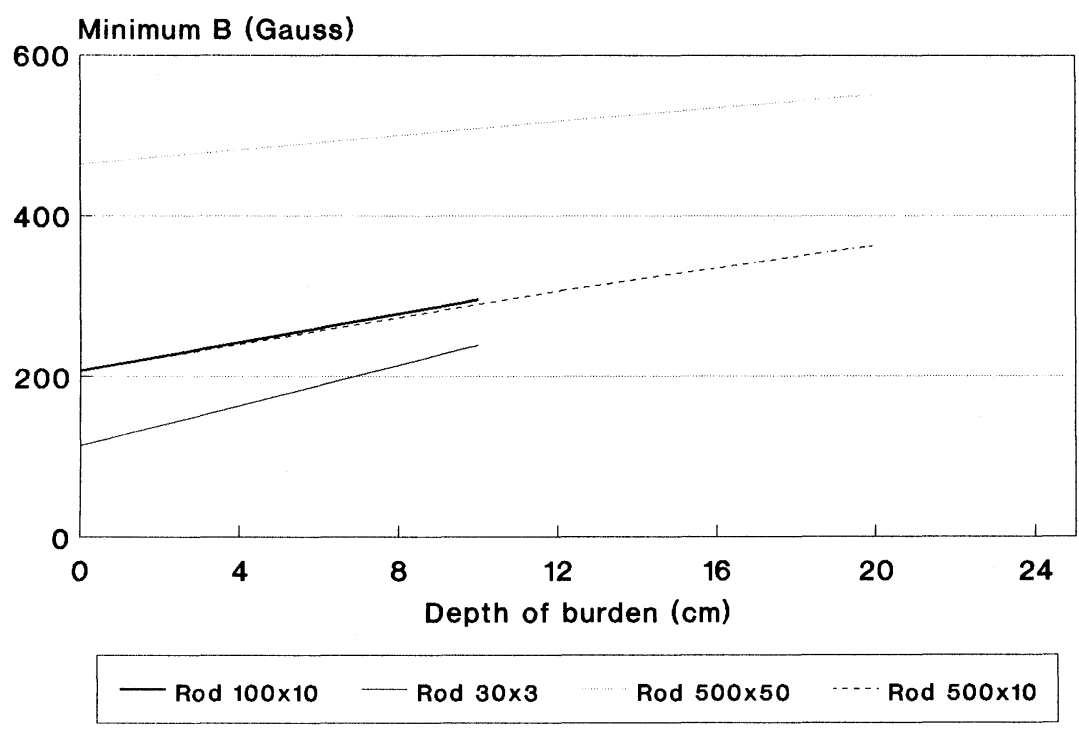

Fig.3. Minimum value of the magnetic field needed to orient steel rods of selected sizes, as a function of the depth of the burden.

In Table II a comparison between theoretical values of $B_{\min }$ calculated using eq. (15), and experimental values is given. It can be seen that agreement is satisfactory. 


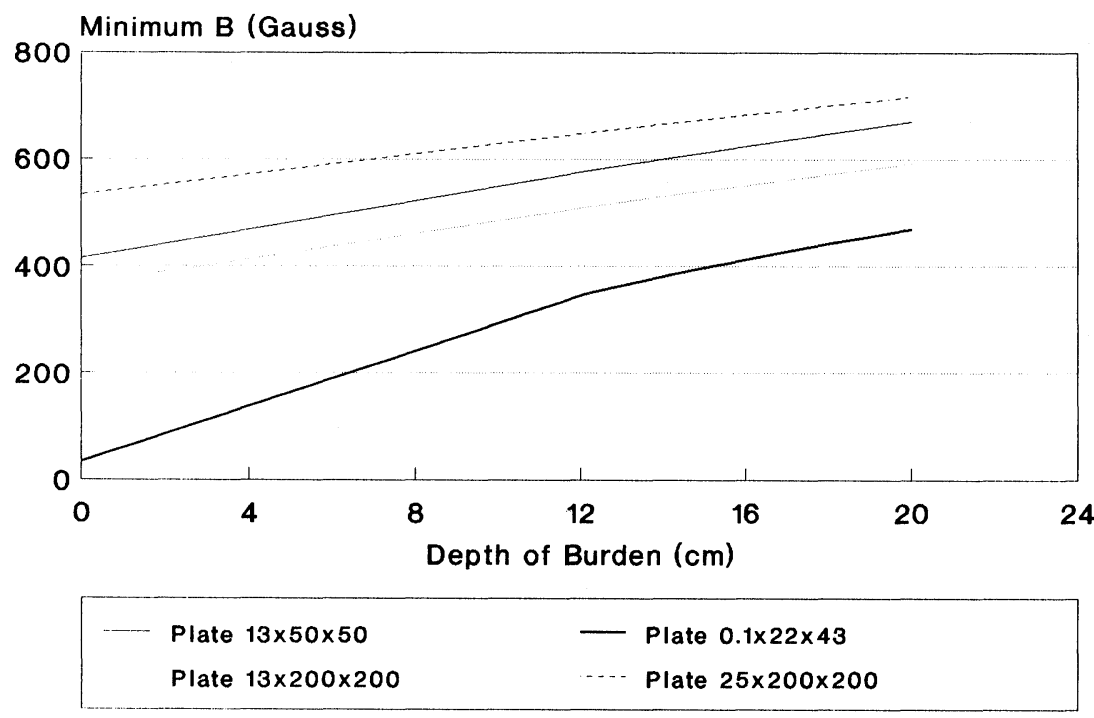

Fig.4. Minimum value of the magnetic field needed to orient steel plates of selected sizes, as a function of the depth of the burden.

\section{Determination of Demagnetization Factors}

The calculation of the demagnetization factor $D$ for objects of shapes different from that of an ellipsoid is complex and the values found in literature are often of limited practical use (ellipsoids, infinite cylinders). Brown [6] gives tables for circular cylinders of finite length, magnetized longitudinally.and for rectangular rods magnetized transversely. These geometries, together with an ellipsoid, should be sufficient for our analysis since other shapes usually encountered in the separation practice (plates, hexagons) can be, in the first approximation, converted into geometries for which the demagnetization factors are available.

Values of demagnetization factors for selected shapes and sizes are given in Table III. 
Table II. Comparison of theoretical and experimental values of minimum magnetic field $B_{\min }$

\begin{tabular}{cccc}
\hline \multirow{2}{*}{ Object } & Burden dept $(\mathrm{mm})$ & Exp. & Theor. \\
\hline Rod & & & \\
$3 \times 30 \mathrm{~mm}$ & 0 & 124 & 113 \\
& 10 & 160 & 132 \\
Plate & & & \\
$0.1 \times 22 \times 43 \mathrm{~mm}$ & 0 & 33 & 34 \\
& 10 & 131 & 114 \\
\hline
\end{tabular}

Table III. Demagnetization factors of ferromagnetic objects of selected shapes and sizes (in SI) [6].

\begin{tabular}{|c|c|c|}
\hline Object & Size & D \\
\hline Sphere & & 0.33 \\
\hline Finite cylin & $\begin{array}{c}\text { (magnetized longitudinally) } \\
\ell / \mathrm{d}=0.0 \\
0.4 \\
1.0 \\
4.0 \\
10.0 \\
40.0 \\
100.0 \\
1000.0\end{array}$ & $\begin{array}{l}1.00 \\
0.528 \\
0.3116 \\
0.098 \\
0.041 \\
0.011 \\
0.004 \\
0.0004\end{array}$ \\
\hline Plate & $\begin{array}{c}\text { (magnetized longitudinally) } \\
13 \times 50 \times 50 \mathrm{~mm} \\
13 \times 200 \times 200 \mathrm{~mm} \\
25 \times 200 \times 200 \mathrm{~mm}\end{array}$ & $\begin{array}{l}0.19 \\
0.06 \\
0.11\end{array}$ \\
\hline
\end{tabular}




\section{The Effect of the Burden}

We have shown that long ferromagnetic objects can be lifted from a conveyor belt most easily when they are first re-orientated in such a way that their long axis (i.e. the axis of the easiest magnetization) is parallel with the direction of the magnetic field.

Once this is achieved, the magnetic force (eq. (1)) is needed to overcome the weight of the object. The object will then be lifted provided eq.(8) is satisfied. In practice, however, a ferromagnetic particle is usually buried in a layer of loosely packed material, e.g. coal. With the body in rest, the minimum magnetic force required to move the object is determined by its weight and by the force of the pressure on the upper hemisphere of the body:

$$
\mathbf{F}_{\mathrm{p}}=\rho_{\mathrm{b}} \mathbf{V}_{\mathrm{b}} \mathbf{g}
$$

where $V_{b}$ is the volume of the burden above the object. It is again assumed that the burden material is such that it does not exert an upward pressure on the lower hemisphere of the object when the magnetic force becomes just large enough to set the object into an upward vertical motion. It is also assumed that the body is already orientated so that its long axis is aligned along the vertical magnetic field. In equilibrium,

$$
\mathbf{F}_{\mathrm{m}}=\mathbf{F}_{\mathrm{g}}+\mathbf{F}_{\mathrm{p}}
$$

From eq.(17), taking into account that $\rho_{\mathrm{p}} \gg \rho_{\mathrm{b}}$, a modified version of eq.(8) can be written:

$$
\mathbf{f}_{\mathbf{m}}=\left[\frac{4 \pi}{\mu_{0}}\right] \frac{\mu_{0}\left[1+\mathrm{D}\left(\mu_{1}-1\right)\right]}{\mu_{1}-1}\left[\rho_{\mathrm{p}}+\rho_{\mathrm{b}} \frac{\mathrm{V}_{\mathrm{b}}}{\mathrm{V}_{\mathrm{p}}}\right] \mathbf{g}
$$

As could have been expected, the static magnetic force density in the presence of the burden depends not only on the shape of the object, but also, in contrast to the situation when the burden is absent (eq.(8)), on the size of the object. Typical dependence of the static magnetic force density on the depth of the burden, for 
various sizes of a ferromagnetic sphere is shown in Fig.5.

Similar diagrams can be constructed, using eq. (18) for rods, plates and other shapes. It can be seen that the static force density increases steeply with increasing depth of the burden for small bodies, while for larger objects the dependence is mild. This is a result of differing relative importance of the volumes of the body and of the the burden, as shown in eq.(18).

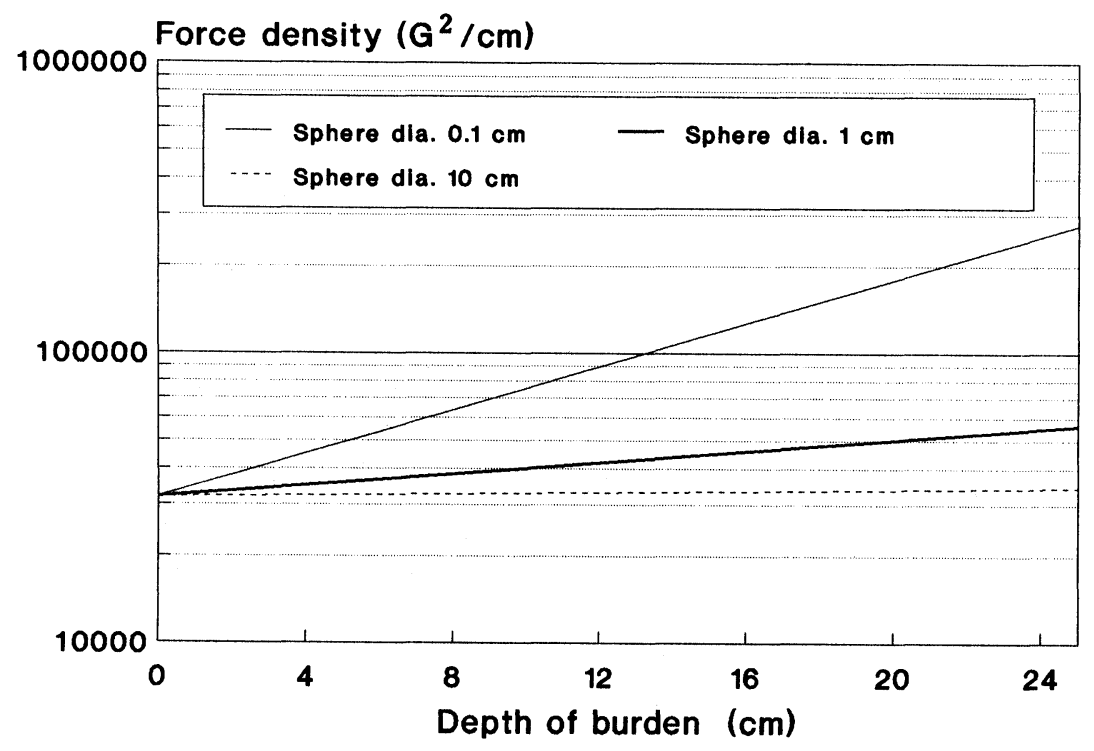

Fig. 5. The static magnetic force density as function of the depth of the burden for various sizes of ferromagnetic spheres. $\mu_{\mathrm{r}}=1000, \rho_{\mathrm{p}}=7.8 \mathrm{gcm}^{-3}, \rho_{\mathrm{b}}=0.8 \mathrm{gcm}^{-3}$.

\section{Movement of a Body Through the Burden}

If the magnetic force density exerted on a ferromagnetic body is large enough to overcome the effect of the weight of the body and the force of friction, the body will start moving upwards under the influence of the external magnetic field.

In view of considerable complexity of the motion of a body through a multiparticle medium, the problem cannot be treated in a universal manner. Although an 
equilibrium dynamic force density for a magnetizable body moving through the burden can be derived, based on the treatment by Barnea and Mizrahi [7], its value for general discussion is limited in view of numerous crude approximations that have to be made.

The treatment of the static situation, as given in previous sections, however, gives a "safe" minimum magnetic field and force density to get the object moving upwards. It is clear that once the object is moving, the equilibrium force required to maintain the motion is smaller than the force applicable to static situation.

\section{THE EVALUATION OF A SUSPENDED MAGNET}

A typical diagram that is often used to evaluate a suspended magnet is shown in Fig. 6. It is a common practice to plot the magnetic induction versus distance from the magnet and then to calculate and plot the force density (force index) as a function of the distance. The force density versus distance curve can then be used to determine the maximum lifting distance from which an object of a given shape will be lifted.

Alternatively, if the lifting distance and the type of object to be lifted are known, one can determine, from Fig. 6, the required force density, and then to design a magnet that would generate such a force density.

Table V Force density on ferromagnetic sphere (diameter $100 \mathrm{~mm}$ ) and rod (diameter $10 \mathrm{~mm}$, length $100 \mathrm{~mm}$ )

\begin{tabular}{lcc}
\hline & & $\mathrm{f}_{\mathrm{m}}\left(\mathrm{G}^{2} \mathrm{~cm}^{-1}\right)$ \\
Force density & Sphere & Rod \\
\hline $\begin{array}{c}\text { Static } \\
\text { (no burden) } \\
\text { Static } \\
\text { (burden } 100 \mathrm{~mm})\end{array}$ & 32000 & 4037 \\
\hline
\end{tabular}


For instance, magnet characterized by the diagram shown in Fig. 6 will be able to lift a sphere from a distance of $435 \mathrm{~mm}$. Similarly, using the standard procedure, the lifting distance of $580 \mathrm{~mm}$ for a steel rod having the length-to-diameter ratio equal to 10 would be determined.

Such a traditional approach ignores the fact that before an object like a rod or a plate can be lifted, it must be re-orientated into such a position where its axis of easy magnetization is .parallel with the direction the magnetic field that induces the lifting action.

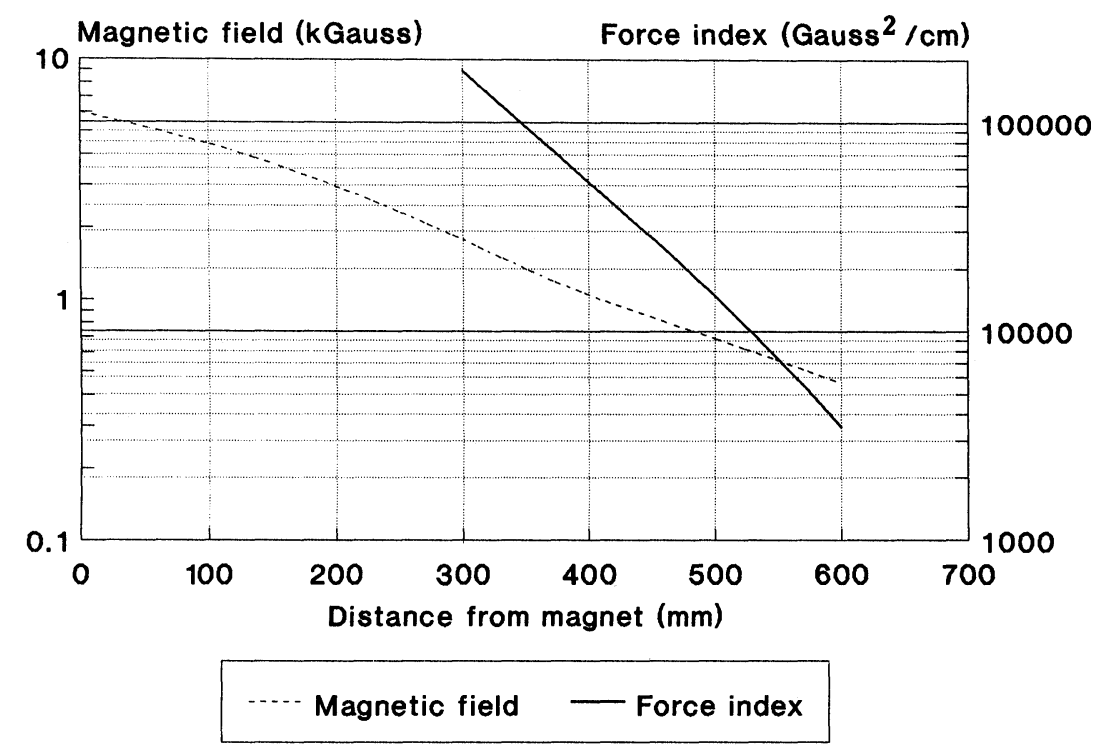

Fig $6 \quad$ Typical diagram used to evaluate a suspended magnet

We have assumed that for such a re-orientation to occur, the magnetic torque exerted on the object must be equal or greater than the torque exerted by the weight of the object and of the burden of material. For such a condition to be met, the force density is irrelevant and the product $B^{2} \sin 2 \alpha$ is decisive.

It is thus clear that for correct evaluation of a suspended magnet the maximum lifting distance based on the minimum magnetic field needed to re-orient must 
be determined. Subsequently, the maximum lifting distance for the body oriented with its axis of easy magnetization along the direction of the magnetic field, based on the force index, will be determined. The smaller of these two distances will be used for the design of the magnet.

A typical example is shown in Fig. 6. It follows from Fig. 4 that in order to re-orient a steel plate $25 \times 200 \times 200 \mathrm{~mm}$, buried in $200 \mathrm{~mm}$ deep layer of burden, the minimum magnetic field of 720 Gauss is needed. It can be seen from Fig. 6 that such a field is obtained at a distance of $500 \mathrm{~mm}$ from the magnet.

The force density required to lift this plate can be determined from eq. (18). If the plate is oriented along the axis of easy magnetization $(D=0.041)$, the force index $f_{\mathrm{m}}=11800 \mathrm{G}^{2} / \mathrm{cm}$ is needed to lift the plate. Fig. 6 shows that such a value of the force index is achieved at a distance of $540 \mathrm{~mm}$ from the magnet. At this distance, however, the magnetic field would be too low to re-orient the body and thus the maximum distance of the belt from the magnet must be $500 \mathrm{~mm}$.

The force index needed to lift the un-oriented plate $(D \cong 0.84)$ is equal to 147250 $\mathrm{G}^{2} / \mathrm{cm}$ which corresponds to the distance of about $250 \mathrm{~mm}$ from the magnet, as shown in Fig. 6.

The most important implication of this approach is that the magnetic field strength and force density must satisfy the required conditions independently. In the traditional approach, the force density, which ascribes to the magnetic field and to the field gradient the same weight factor, is used as the only criterion which may result in erroneous design of the magnetic system, by underdesigning the magnetic field strength and overdesigning the field gradient.

\section{ACKNOWLEDGEMENT}

The author wishes to thank Dr. V. Hnizdo, Department of Physics, University of the Witwatersrand, Johannesburg, South Africa for numerous valuable discussions and suggestions. 


\section{NOMENCLATURE}

a dimension of a particle

b dimension of a particle

B magnetic induction

d particle diameter

D demagnetization factor in SI

$f_{m} \quad$ magnetic force density

$\mathrm{F}_{\mathrm{g}} \quad$ force of gravity

$\mathrm{F}_{\mathrm{m}}$ magnetic force

$\mathrm{F}_{\mathrm{p}}$ force of pressure

$\mathrm{g}$ acceleration of gravity

$\mathrm{h}$ height of burden

$\mathrm{H}$ magnetic field strength

m effective magnetic dipole moment

M magnetization of the body

$\mathrm{V}$ volume

$\mathrm{V}_{\mathrm{b}} \quad$ volume of burden

$\mathrm{V}_{\mathrm{p}} \quad$ volume of particle

$t$ thickness of particle

$\mathrm{T}$ magnetic torque

$\alpha \quad$ angle between the direction of $B_{0}$ and the axis of symmetry of the body

$\mu_{0} \quad$ magnetic permeability of vacuum

$\mu_{1} \quad$ relative magnetic permeability of magnetizable body

$\mu_{2} \quad$ relative magnetic permeability of external medium

$\rho_{\mathrm{b}} \quad$ bulk density of burden

$\rho_{\mathrm{f}} \quad$ density of multiparticle system

$\rho_{\mathrm{m}} \quad$ density of medium

$\rho_{\mathrm{p}} \quad$ density of particle

\section{REFERENCES}

[1] A manual published by Magnetic Equipment Standards Council, U.S.A. (The date and place of publication unknown)

[2] A.W. Hamilton, E.S. Twichell, EMJ, September 1971, p. 142

[3] F.J. Lowes, Proc. Roy. Soc. London A337(1974), 555

[4] R.W. Whitworth, H.V. Stopes-Roe, Nature 234 (1971), 31

[5] L.D. Landau and E.M. Lifshitz: Electrodynamics of Continuous Media. Pergamon Press, Oxford 1984, $2^{\text {nd }}$ Edition, p. 42

[6] W.F. Brown, Jr.: Magnetostatic Principles in Ferromagnetism, North-Holland Publ. Co., Amsterdam 1962

[7] E. Barnea, J. Mizrahi; Chem. Eng. J. 5 (1973), 171

Keywords: suspended magnet, force index, force density, magnetic force, magnetic torque, demagnetization factor 